\title{
Surgical excision of osteochondroma on mandibular condyle via preauricular approach with zygomatic arch osteotomy
}

\author{
Sang-Hoon Park, Jun-Hyeong An, Jeong Jun Han, Seunggon Jung, Hong-Ju Park, Hee-Kyun Oh
} and Min-Suk Kook ${ }^{*}$ (1)

\begin{abstract}
Background: Osteochondroma is a benign tumor that tends to develop in mandibular condyle and coronoid process in the craniofacial region. If tumor mass has grown from condyle into the infratemporal space with zygomatic arch obstructing the access, there are risks associated with surgical exposure and local resection of these masses.

Case presentation: This study reports on a case of osteochondroma on mandibular condylar head where we treated with surgical excision via preauricular approach with 3D analysis. After the local resection, there were no surgical and post-operative complications until 8-month follow-up period.
\end{abstract}

Conclusions: In local excision of osteochondroma, our method is a minimally invasive method. It is a good example of osteochondroma treatment.

Keywords: Osteochondroma, 3D computer modeling, Minimally invasive

\section{Background}

Osteochondroma is characterized by cartilage-capped osseous protrusion from the external surface of the affected bone [1]. It can potentially occur at any site of endochondral ossification. In the craniofacial region, mandibular condyle and coronoid process are the most common affected sites. Some authors have classified this tumor in mandibular condyle into two types: type 1 , a protruding expansion with mean proliferation direction of mass in one way, and type 2, a globular expansion with mean mass proliferate in all directions (Fig. 1) [2]. Almost all osteochondroma in mandibular condyle is type 1 [2]. The present case was also type 1 .

If tumor mass has grown into the infratemporal space with zygomatic arch obstructing the access, there are risks associated with surgical exposure and local resection of these masses [3]. To reduce such risks, we visualized tumor mass and surrounding structures using 3D computer modeling program. With 3D CT work-up,

\footnotetext{
*Correspondence: omskook@jnu.ac.kr

Department of Oral and Maxillofacial Surgery, School of Dentistry, Dental Science Research Institute, Chonnam National University, 77, Yongbongro, Buk-Gu, Gwangju 500-757, Korea
}

Springer Open

(c) The Author(s). 2017 Open Access This article is distributed under the terms of the Creative Commons Attribution 4.0 International License (http://creativecommons.org/licenses/by/4.0/), which permits unrestricted use, distribution, and reproduction in any medium, provided you give appropriate credit to the original author(s) and the source, provide a link to the Creative Commons license, and indicate if changes were made.

\section{Case presentation}

A 46-year-old man was referred to our clinic due to chief complaints of chin deviation to the right side. There were right posterior cross bite and dental midline deviation to the right side by $5 \mathrm{~mm}$ (Fig. 2). In panorama 


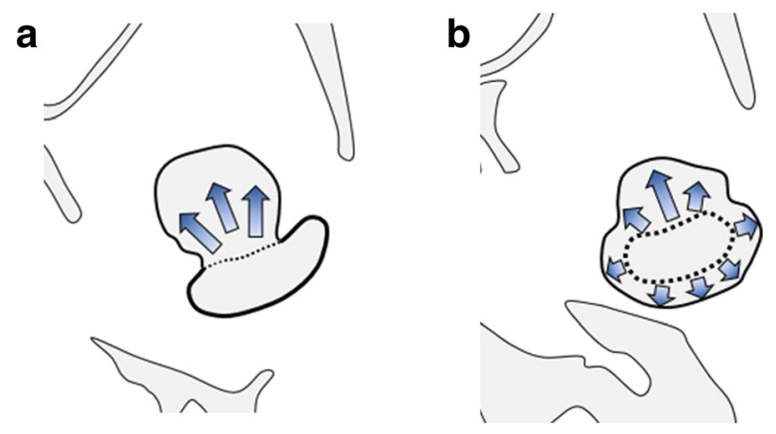

Fig. 1 Classifications of osteochondroma. a Type 1 osteochondromaprotruding expansion. b Type 2 osteochondroma-globular expansion

radiograph, bony exophytic proliferation of the condyle was observed (Fig. 3). With additional imaging of CT and three-phase bone scan, tentative diagnosis of osteochondroma or osteoma on the left mandibular condyle was made.

\section{D CT work-up}

Reconstruction and differentiation of tumor mass and surrounding structures were done using Mimics (Materialize NV, Leuven, Belgium). In a mandible only view, tumor mass could be observed at all directions. Resection line of tumor mass was identified (Fig. 4). We could anticipate that zygomatic arch obstructed access to the resection line and removal of the resected mass; thus, we plan zygomatic arch osteotomy. The tumor mass was about $20 \times 20 \times$ $15 \mathrm{~mm}$; the size of zygomatic arch osteotomy was enough to be $15 \mathrm{~mm}$ for drawing out the resected mass.

\section{Surgical procedure}

Under general anesthesia, a preauricular incision was made. Dissection and bleeding control was done until zygomatic arch and temporomandibular joint capsule appeared. Two osteotomy lines were made. A posterior

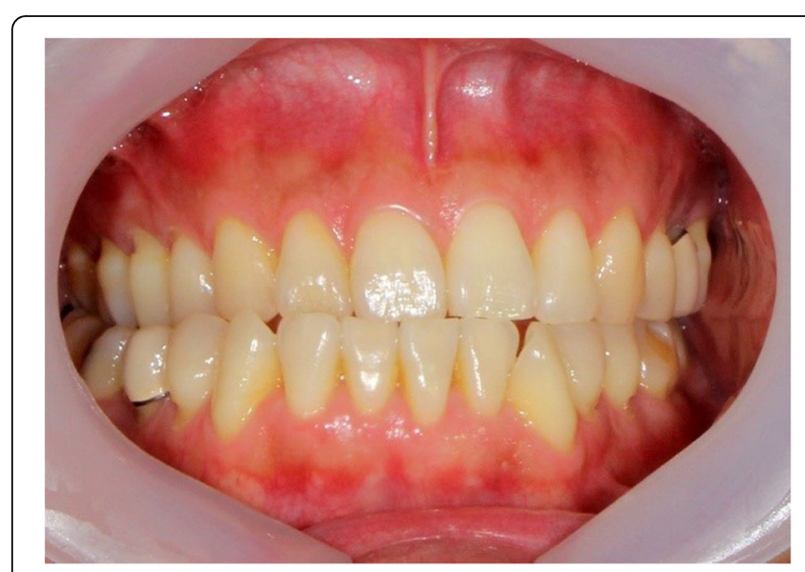

Fig. 2 Menton is deviated to the right side. Dental midline is shifted to the right side

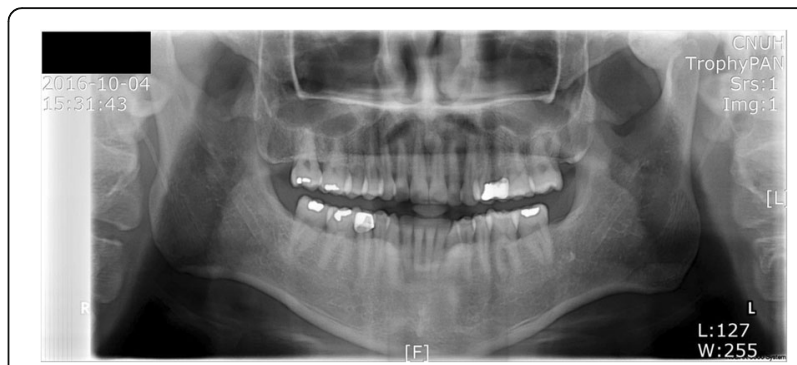

Fig. 3 Proliferation of the left condylar head could be easily checked

one was made at the foremost point of articular eminence while an anterior one was made at $15 \mathrm{~mm}$ in front of the posterior line. Zygomatic arch osteotomies were completed, and the fragment was displaced downward with the masseter muscle still attached. The joint capsule was incised, and the lateral pterygoid muscle was dissected to expose the tumor mass. Tumor mass was resected with saw. The surface of condyle was smoothed with round bur. Tumor mass was sent to the Department of Pathology. The zygomatic arch fragment was repositioned and fixed with a four-hole straight shape miniplate (Fig. 5). Finally, normal occlusion and proper midline relationship were checked (Fig. 6).

After the operation, intermaxillary fixation was done for 2 weeks with rubber rings. A month later, normal occlusion with proper dental midline was maintained (Fig. 6).

CT was taken immediately after the operation. It confirmed that the tumor mass was removed (Fig. 7).

Pathologic diagnosis of the tumor mass was the osteochondroma (Fig. 8).

\section{Discussion}

There are many theories about the etiology and pathogenesis of cartilage-capped and exophytic bony growths. They can occur in bones formed by endochondral ossification. They can also develop from displacement of the

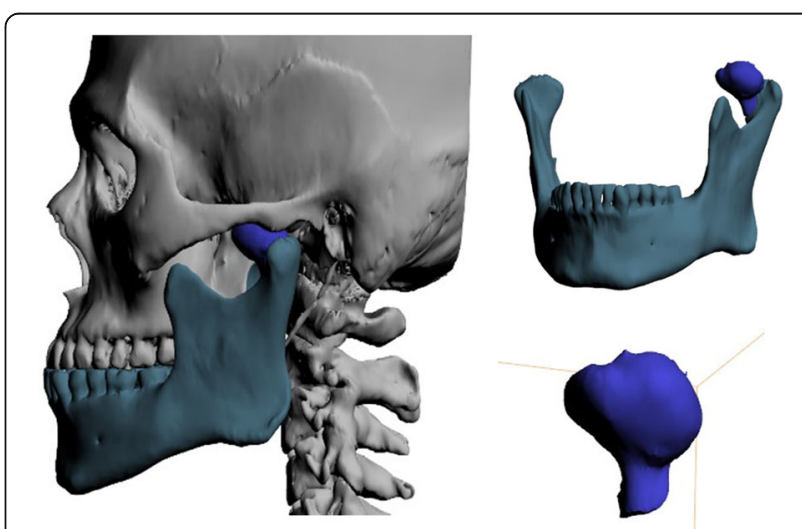

Fig. 4 Reconstruction of three-dimensional image was done 

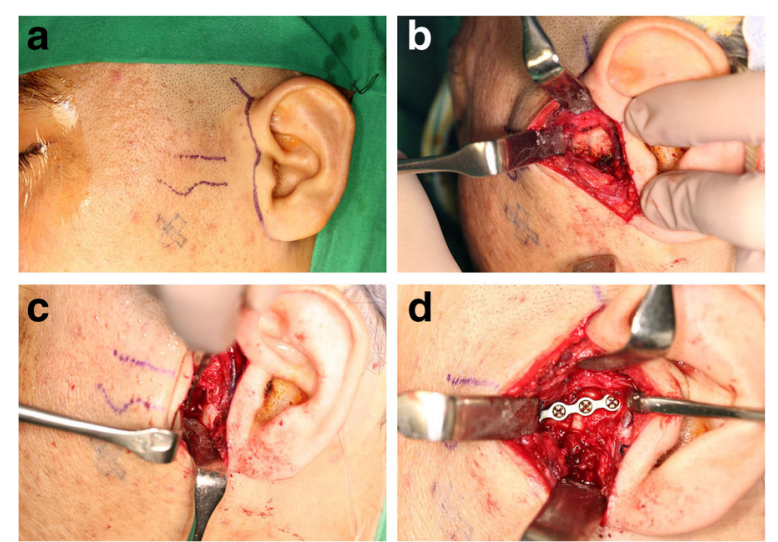

Fig. 5 a Drawing of incision line. b Exposure of zygomatic arch. c Osteotomy of zygomatic arch. d Reposition of the zygomatic arch fragment after resection of tumor mass

lateral portion of the growth plate which then proliferates in a direction diagonal to the long axis of the bone and away from the nearby joint [8]. In our patient, exophytic projection was extruded from the lateral portion of the condyle head with a direction diagonal to the long axis of the condyle. The peripheral part of the physis has been considered as a hernia from the growth plate [9]. This hernia may be idiopathic or traumatic. Regardless of the cause, the result is an abnormal extension of metaplastic cartilage responding to factors that cause exostosis growth by stimulating the growth plate. Development of these tumors in the mandibular condyle tends to support the theory that epiphyseal cartilage is idly located on the bone surface. It has been suggested that stress in the tough insertion region where local accumulation of cartilage dislocation is present can induce the formation of these tumors [2]. This could explain the fact that in the mandible, these lesions often arise at the coronoid process attached to temporalis and anteromedial condylar region attached to lateral pterygoid muscle insertion. Some authors also believe that trauma might play a role in the formation of these lesions [1].

Histopathological findings are crucial to the diagnosis of osteochondroma. Histologic examinations include a cartilaginous cap similar to that seen in a normal cartilage, endochondral ossification, cartilaginous islands in the subcortical bone, and a marrow space contiguous with the underlying bone (Fig. 8). It has been reported that the cartilaginous cap might be $10 \mathrm{~mm}$ or greater in thickness in the axial skeleton [10]. However, it tends to be thinner in the maxillofacial region. It might be absent in long-standing cases [10].

When considering treatment, the growing state and the type of osteochondroma are crucial. Growth status can be judged by repeated occlusal evaluation or bone scintigraphy. If an active growth is noticed in a child and the asymmetry is large, subtotal condylectomy is usually performed. However, in adult patient with the same symptoms, both side condylectomy and orthognathic surgery should be included. After such condylectomy, lateral open bite on the contralateral side might occur unless some kind of reconstruction is performed. A costochondral graft or a total stock joint prosthesis may be used. However, it has disadvantages such as exploration of the second surgical site, donor site morbidity, and bone resorption. A total joint prosthesis has disadvantages of high cost, material wear and potential failure, and restricted use in the growing patient $[11,12]$. An

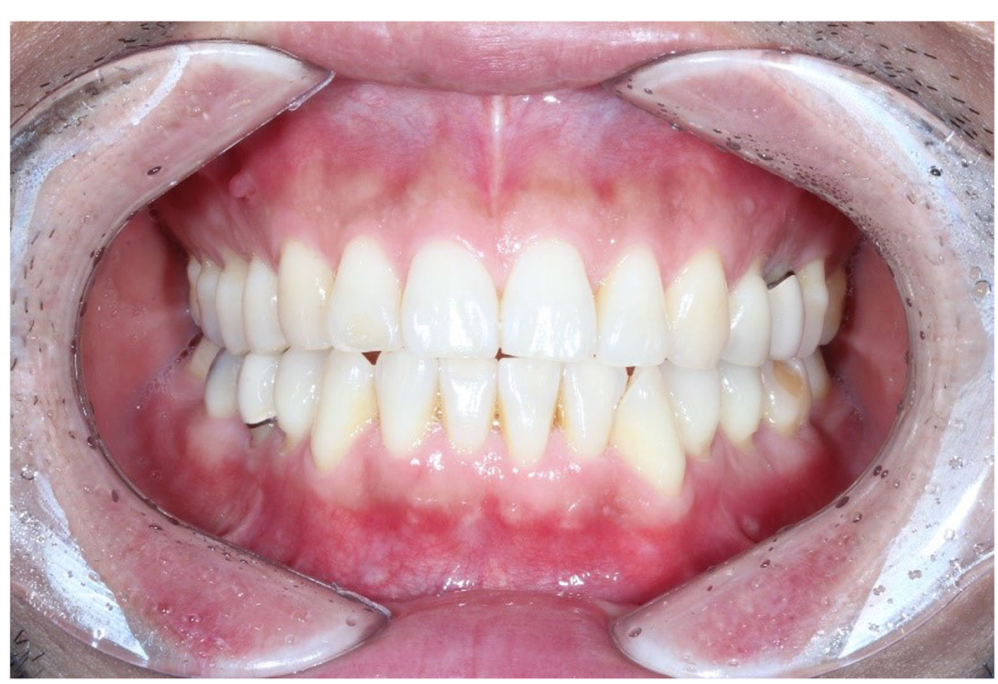

Fig. 6 Dental midline is corrected after the surgery 


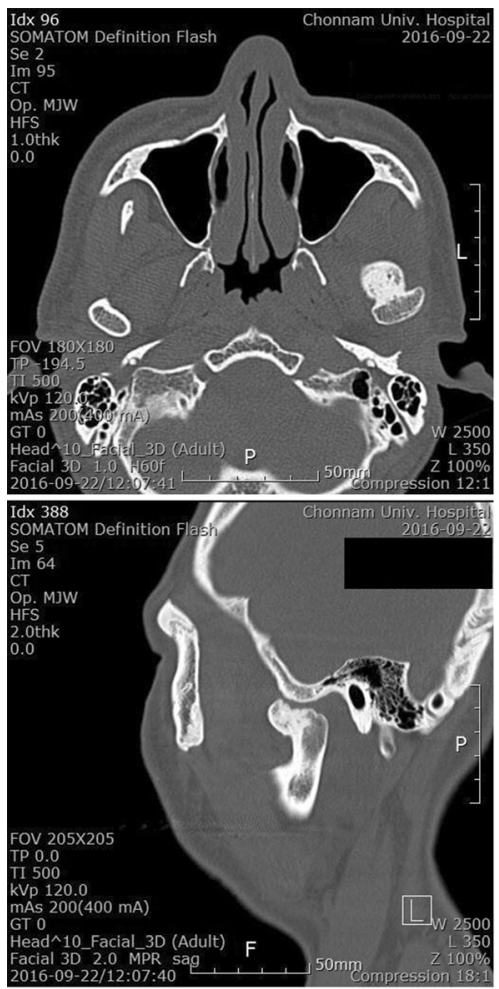

Preoperative

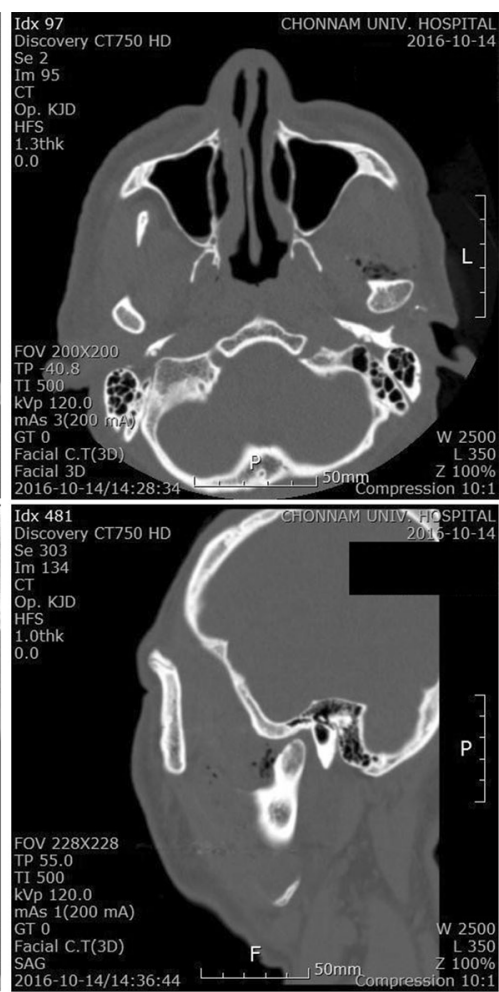

Postoperative

Fig. 7 Preoperative CT scan (left) and postoperative CT scan (right)

alternative method might be vertical osteotomy of the ramus and advancing it superiorly to form a new condyle underneath the disc as described previously [13]. Locally derived bone graft attached to the medial pterygoid muscle has been utilized [14]. Some authors have proposed conservative condylectomy with less

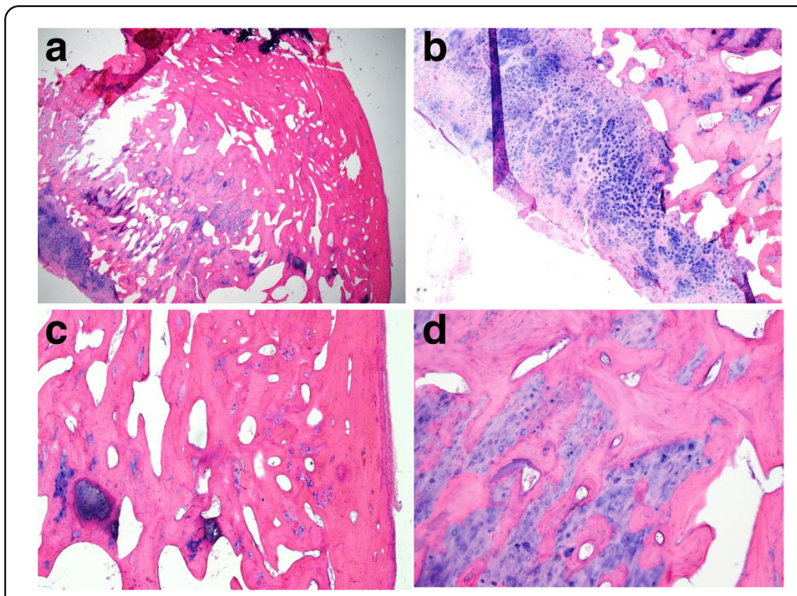

Fig. 8 Histopathologic findings of the tumor mass. a $\times 10$, cartilaginous cap could be identified. $\mathbf{b} \times 20$, head of the condyle. c $\times 40$, endochondral ossification progressing beneath the cartilaginous cap. $\mathbf{d} \times 100$, hyaline cartilage gradually changed into trabecular bone complications [15]. This protocol is applicable to osteochondromas involving the head of the condyle without extension of tumor into the neck. In patients with osteochondroma, the condylar head usually enlarges fairly and the neck of the condyle is significantly thickened [13]. Such neck thickening makes it possible to reproduce the remaining condylar stump for functioning as a "new" condylar head. The articular disc is then repositioned onto the "new" condyle and stabilized [13].

If the condition is inactive and there is no TMJ symptom, the reason for surgical intervention can be cosmetic or associated with dysfunction of mastication. In this situation, treatment can be chosen depending on osteochondroma classification. In type 2 with globular expansion, condylectomy should be performed as described above. In type 1 with protruding expansion, just local excision is sufficient. Some excellent outcomes have been reported for type 1 [12]. In this study, the patient's age was 37 years old. There was a mild bone activity on the condyle head in the three-phase bone scan. Exophytic bony protrusion area was approximately $2 \times$ 2 - $\mathrm{cm}$ sized, heading for anteromedial of the condyle. It was type 1 osteochondroma. Based on these facts, we decided to do local excision.

After 3D reconstruction of the CT images, the tumor mass was growing to anteromedial side of the 
mandibular condyle; thus, it might be difficult to approach to the mass through only preauricular incision due to the obstruction of zygomatic arch (Fig. 4). To allow the access of cutting instruments to the anteromedial side of the mandibular condyle and removal of the excised tumor without extension of incision or dissection, we planned zygomatic arch osteotomy over the tumor mass. During the surgery, zygomatic arch osteotomy provided easy access to anteromedial portion of the condyle without additional incision or wide dissection, and tumor mass was drawn out with only $4 \mathrm{~cm}$ of preauricular incision with zygomatic arch osteotomy. Though minimally invasive procedure in this study cannot be applied in all osteochondroma cases, it can be one of the simple treatments for not-growing type 1 osteochondromas.

The malignant potential and risk of recurrence are the major drawbacks of conservative procedure. The recurrence rate for solitary osteochondromas in long bone is approximately $2 \%$ [16]. Of all the condylar osteochondromas reported, three have shown recurrence $[15,17,18]$. For the three cases, excision and condyloplasty rather than condylectomy were done as the initial procedure [1]. Because of a short follow-up period, we cannot evaluate recurrence for this case. However, based on mild bone activity in three-phase bone scan and other cases in the literature, it is expected that there will be no recurrence.

\section{Conclusions}

Osteochondroma in the facial area is an uncommon disease. However, more cases have been reported and its treatment has been systematized. Its growth state and the type of tumor are important to decide treatments. In the case of inactive type 1 osteochondroma, local excision alone can provide good results. In local excision of osteochondroma, our method is a minimally invasive method. It is a good example of osteochondroma treatment.

\section{Acknowledgements \\ None. \\ Funding \\ No funding was received. \\ Availability of data and materials \\ Not applicable.}

\section{Authors' contributions}

PSH wrote the manuscript. AJH, JJH, and SGJ helped in drafting the manuscript. $\mathrm{HJP}$ and HKO were involved in revising the manuscript. MSK carefully reviewed and revised the manuscript. All authors read and approved the final manuscript.

\section{Consent for publication}

Written informed consent was obtained from the patient for publication of this case report and accompanying images.

\section{Competing interests}

The authors declare that they have no competing interests.

\section{Publisher's Note}

Springer Nature remains neutral with regard to jurisdictional claims in published maps and institutional affiliations.

Received: 17 July 2017 Accepted: 13 September 2017

Published online: 25 October 2017

\section{References}

1. Roychoudhury A, Bhatt K, Yadav R (2011) Review of osteochondroma of mandibular condyle and report of a case series. J Oral Maxillofac Surg 11:2815-2823

2. Chen MJ, Yang C, Qiu YT (2014) Osteochondroma of the mandibular condyle: a classification system based on computed tomographic appearances. J Craniofac Surg 5:1703-1706

3. Chotai S, Kshettry VR, Petrak A (2015) Lateral transzygomatic middle fossa approach and its extensions: surgical technique and 3D anatomy. Clin Neurol Neurosurg 130:33-41

4. Saito T, Utsunomiya T, Furutani M (2001) Osteochondroma of the mandibular condyle: a case report and review of the literature. J Oral Sci 4:293-297

5. Aydin MA, Kucukcelebi A, Sayilkan S (2001) Osteochondroma of the mandibular condyle: report of 2 cases treated with conservative surgery. J Oral Maxillofac Surg 9:1082-1089

6. Cottrell DA (2002) Use of conservative condylectomy for treatment of osteochondroma of the mandibular condyle-discussion. J Oral Maxillofac Surg 3:268

7. Rivera H, Bastidas R, Acevedo AM (1998) A conservative surgical approach of osteochondroma affecting the mandibular condyle. Investig Clin 2:117-124

8. Kitsoulis P, Galani V, Stefanaki K (2008) Osteochondromas: review of the clinical, radiological and pathological features. In Vivo 5:633-646

9. D'Ambrosia R, Ferguson AB Jr (1968) The formation of osteochondroma by epiphyseal cartilage transplantation. Clin Orthop Relat Res 61:103-15

10. Schajowicz F (1981) Tumors and tumor-like lesions of bone and joints. Springer-Verlag New York, New York xiv, 581 p., 2 leaves of plates

11. Song D, Zhu S, Hu J (2009) Use of ramus osteotomy for the treatment of osteochondroma in the mandibular condyle. J Oral Maxillofac Surg 3:676-680

12. Ramos-Murguialday M, Morey-Mas MA, Janeiro-Barrera S (2012) Osteochondroma of the temporomandibular joint: report of 2 cases emphasizing the importance of personalizing the surgical treatment. Oral Surg Oral Med Oral Pathol Oral Radiol 3:e41-e47

13. Loftus MJ, Bennett JA, Fantasia JE (1986) Osteochondroma of the mandibular condyles. Report of three cases and review of the literature. Oral Surg Oral Med Oral Pathol 3:221-226

14. Holmlund AB, Gynther GW, Reinholt FP (2004) Surgical treatment of osteochondroma of the mandibular condyle in the adult. A 5-year followup. Int J Oral Maxillofac Surg 6:549-553

15. Wolford LM, Mehra P, Franco P (2002) Use of conservative condylectomy for treatment of osteochondroma of the mandibular condyle. J Oral Maxillofac Surg 3:262-268

16. Seki H, Fukuda M, Takahashi T (2003) Condylar osteochondroma with complete hearing loss: report of a case. J Oral Maxillofac Surg 1:131-133

17. Peroz I, Scholman HJ, Hell B (2002) Osteochondroma of the mandibular condyle: a case report. Int J Oral Maxillofac Surg 4:455-456

18. Vezeau PJ, Fridrich KL, Vincent SD (1995) Osteochondroma of the mandibular condyle: literature review and report of two atypical cases. J Oral Maxillofac Surg 8:954-963

\section{Submit your manuscript to a SpringerOpen ${ }^{\mathcal{O}}$ journal and benefit from:}

- Convenient online submission

- Rigorous peer review

- Open access: articles freely available online

- High visibility within the field

- Retaining the copyright to your article

Submit your next manuscript at $>$ springeropen.com 